\title{
Some strange behaviors of the power series ring $R[[X]]$
}

\author{
Phan Thanh Toan ${ }^{1, *}$ \\ ${ }^{1}$ Fractional Calculus, Optimization and Algebra Research Group, Faculty of Mathematics and Statistics, \\ Ton Duc Thang University, Ho Chi Minh City, Vietnam
}

\begin{abstract}
Let $R$ be a commutative ring with identity. Let $R[X]$ and $R[[X]]$ be the polynomial ring and the power series ring respectively over $R$. Being the completion of $R[X]$ (under the $X$-adic topology), $R[[X]]$ does not always share the same property with $R[X]$. In this paper, we present some known strange behaviors of $R[[X]]$ compared to those of $R[X]$.
\end{abstract}

\section{Introduction}

In this paper, a ring means a commutative ring with identity. Let $R$ be a ring. Let $R[X]$ and $R[[X]]$ be the polynomial ring and the power series ring respectively over $R$. Being the completion of $R[X]$ (under the $X$-adic topology), $R[[X]]$ does not always share the same property with $R[X]$. In this paper, we present some strange behaviors of $R[[X]]$ compared to those of $R[X]$. Aspects of $R[[X]]$ that will be considered include Krull dimension, transcendence degree, and Noetherian property.

Let $R$ be a ring. If there exists a chain $P_{0} \subset P_{1} \subset \cdots \subset P_{n}$ of $n+1$ prime ideals of $R$, but no such chain of $n+2$ prime ideals, then we say that the Krull dimension of $R$ is $n$ (or $R$ is $n$-dimensional) and write Krull-dim $R=n$. Otherwise, we say that the Krull dimension of $R$ is infinite (or $R$ is infinite-dimensional) and write $\operatorname{Krull-\operatorname {dim}} R=\infty$. For a cardinal number $\alpha$, we say that $\operatorname{dim} R=\alpha$ if $R$ has a chain of prime ideals with length $\alpha$ but no longer chains. (The length of a chain $\mathcal{P}$ of prime ideals of $R$ is defined by $|\mathcal{P}|-1$, where $|\mathcal{P}|$ denotes the cardinality of $\mathcal{P}$. For two chains $\mathcal{P}$ and $Q$ of prime ideals, we say that $\mathcal{P}$ is longer than $Q$ if $|\mathcal{P}|>|Q|)$. We also say that $\operatorname{dim} R \geq \alpha$ if there is a chain of prime ideals of $R$ with length $\geq \alpha$ and that $\operatorname{dim} R \leq \alpha$ if every chain of prime ideals of $R$ has length $\leq \alpha$. Hence, $\operatorname{dim} R=\alpha$ if and only if $\operatorname{dim} R \geq \alpha$ and $\operatorname{dim} R \leq \alpha$. We note that if $\operatorname{Krull}-\operatorname{dim} R=n<\infty$, then $\operatorname{dim} R=$ Krull-dim $R$. Furthermore, $\operatorname{dim} R \geq \boldsymbol{\aleph}_{0}$ implies Krull-dim $R=\infty$ but not vice versa.

The Krull dimension of the polynomial ring $R[X]$ is fairly well-known for a finitedimensional ring $R$. For example, $\operatorname{dim} R\left[X_{1}, \ldots, X_{n}\right]=\operatorname{dim} R+n$ if $R$ is a Noetherian ring or a Prüfer domain [16, 17]. In general, $\operatorname{dim} R+1 \leq \operatorname{dim} R[X] \leq 2 \operatorname{dim} R+1$ [16]. For the power series ring $R[[X]]$, it is shown by J.T. Arnold that $\operatorname{dim} R[[X]] \geq \boldsymbol{\aleph}_{0}$ if $R$ is a non-SFT ring. He defines an ideal $I$ of a ring $R$ to be an SFT ideal if there exist a finitely generated ideal $J \subseteq I$ and $k \in \mathbb{N}$ such that $a^{k} \in J$ for each $a \in I$ and he calls a ring $R$ an SFT ring if every ideal of $R$ is an SFT ideal. Typical examples of non-SFT domains are finite-dimensional nondiscrete valuation domains and non-Noetherian almost Dedekind domains. Kang and Park [9] showed that $\operatorname{dim} V[[X]] \geq|\mathbb{R}|$ and hence $\operatorname{dim} V[[X]] \geq \boldsymbol{\aleph}_{1}$ for a 1-dimensional nondiscrete

\footnotetext{
*e-mail: phanthanhtoan@tdtu.edu.vn
} 
valuation domain $V$. Kang and Toan [12] proved that $\operatorname{dim} V[[X]] \geq 2^{\aleph_{1}}$ for a 1-dimensional nondiscrete valuation domain $V$. Loper and Lucas $[14,15]$ also achieved the result that $\operatorname{dim} D[[X]] \geq 2^{\aleph_{1}}$ for a non-Noetherian almost Dedekind domain or a 1-dimensional nondiscrete valuation domain $D$. Recently, Toan and Kang [11] generalize the above results by showing that $\operatorname{dim} R[[X]] \geq 2^{\aleph_{1}}$ if $R$ is a non-SFT domains. In Section 2 , we are going to introduce their technique in proving this result.

Let $D$ be an integral domain with quotient field $K$. It is easy to see that the polynomial rings $D[X]$ and $K[X]$ have the same quotient field. However, as mentioned in [3], it is rare that $D[[X]]$ and $K[[X]]$ have the same quotient field. Except for the trivial case when $D$ is a field, the only example showing that $D[[X]]$ and $K[[X]]$ have the same quotient field is given by Gilmer in [7]. For two integral domains $D_{1} \subseteq D_{2}$, denote by $\operatorname{tr} . d .\left(D_{2} / D_{1}\right)$ the transcendence degree of the quotient field of $D_{2}$ over that of $D_{1}$. Hence, for a cardinal number $\alpha$, tr.d. $\left(D_{2} / D_{1}\right) \geq \alpha$ if there exists a subset of the quotient field of $D_{2}$ with cardinality at least $\alpha$ that is algebraically independent over the quotient field of $D_{1}$. Sheldon showed in [18] that if $D$ contains a nonzero element $a$ such that $\cap_{i=1}^{\infty} a^{i} D=(0)$, then $\operatorname{tr} . d .(D[[x / a]] / D[[X]]) \geq \boldsymbol{\aleph}_{0}$ and hence $\operatorname{tr} . d .(K[[X]] / D[[X]]) \geq \boldsymbol{\aleph}_{0}$ since $D[[x / a]] \subseteq D[1 / a][[X]] \subseteq K[[X]]$. In [2], Arnold and Boyd made a great improvement of this result by showing that if $K[[X]]$ and $D[[X]]$ have different quotient fields, then $\operatorname{tr} . d .(K[[X]] / D[[X]]) \geq \boldsymbol{\aleph}_{0}$. We present here the technique in [8], where the authors showed that whenever $K[[X]]$ and $D[[X]]$ have different quotient fields, $\operatorname{tr} . d .(K[[X]] / D[[X]]) \geq \aleph_{1}$. Note that in general the bound $\aleph_{1}$ is the greatest lower bound that one can obtain since under the continuum hypothesis the cardinality of the quotient field of $K[[X]]$ is exactly $\boldsymbol{\aleph}_{1}$ provided that $K$ is countable.

Let $\alpha$ be an infinite cardinal number (e.g., $\alpha=\boldsymbol{\aleph}_{0}, \boldsymbol{\aleph}_{1}, \ldots$ ). An ideal $I$ of a ring $R$ is called an $\alpha$-generated ideal if $I$ can be generated by a set with cardinality $\leq \alpha . R$ is called an $\alpha$ generated ring if every ideal of $R$ is an $\alpha$-generated ideal. By definition, an $\boldsymbol{\aleph}_{0}$-generated ring is a ring whose ideals are countably generated. Trivial examples of $\boldsymbol{\kappa}_{0}$-generated rings are those that have only countably many elements (so that each ideal has itself as a countable generating set). Every Noetherian ring is obviously an $\boldsymbol{\aleph}_{0}$-generated ring. However, the converse does not hold. Polynomial rings $R\left[X_{1}, X_{2}, \ldots, X_{n}, \ldots\right]$ in countably infinite indeterminates over countable rings $R$, the ring $O$ of algebraic integers, the $\operatorname{ring} \operatorname{Int}(\mathbb{Z})$ of integer-valued polynomials on $\mathbb{Z}$, and 1-dimensional nondiscrete valuation domains are good examples of $\boldsymbol{\kappa}_{0}$-generated rings that are not Noetherian rings.

Even though the class of $\boldsymbol{\aleph}_{0}$-generated rings is strictly larger than the class of Noetherian rings, it is shown in [10] that when restricted to power series rings, they are actually the same. In other words, the concepts " $\boldsymbol{\aleph}_{0}$-generated ring" and "Noetherian ring" are the same for the power series ring $R \llbracket X \rrbracket$. This shows a strange behavior of the power series ring $R \llbracket X \rrbracket$ compared to that of the polynomial ring $R[X]$. Indeed, for any infinite cardinal number $\alpha$, $R$ is an $\alpha$-generated ring if and only if $R[X]$ is an $\alpha$-generated ring, which is an analogue of Hilbert Basis Theorem stating that $R$ is a Noetherian ring if and only if $R[X]$ is a Noetherian ring.

\section{Krull dimension of $R[[X]]$}

\subsection{Krull dimension of $R[X]$}

The aim of this section is to show that $\operatorname{dim} R[[X]] \geq 2^{\aleph_{1}}$ if $R$ is a non-SFT domain, which contrasts with the fact that the Krull dimension of the polynomial ring $R[X]$ is always finite provided that $R$ is. The following result is from [16, Theorems 2 and 9].

Theorem 2.1 If $R$ is a finite-dimensional ring, then

$$
\operatorname{dim} R+1 \leq \operatorname{dim} R[X] \leq 2 \operatorname{dim} R+1 .
$$


Furthermore, if $R$ is a Noetherian ring, then $\operatorname{dim} R[X]=\operatorname{dim} R+1$.

When $R$ is a Prüfer domain, one has the following.

Theorem 2.2 ([17, Theorem 4]) If $R$ is a finite-dimensional Prüfer domain, then

$$
\operatorname{dim} R\left[X_{1}, \ldots, X_{n}\right]=\operatorname{dim} R+n .
$$

\subsection{Construction of an $\eta_{1}$-set $\mathcal{A}$}

We first construct an $\eta_{1}$-set $\mathcal{A}$. This $\eta_{1}$-set will be the index set of an infinite chain of prime ideals in $D \llbracket X \rrbracket$ when $D$ is a non-SFT domain.

- Let $\mathbb{N}=\{1,2,3, \ldots\}$ be the set of positive integers and let $\mathcal{U}$ be the set of all subsets $U$ of $\mathbb{N}$ such that $U=\{n, n+1, \ldots\}$ for some $n \in \mathbb{N}$.

- For two sequences of positive integers $s=\left\{s_{n}\right\}, t=\left\{t_{n}\right\}$, define $s \gg t$ (or $t \ll s$ ) if for each positive integer $k$, there is a set $U \in \mathcal{U}$ (depending on $k$ ) such that $s_{n}>k t_{n}$ for each $n \in U$, i.e., $s_{n}>k t_{n}$ for all large $n$.

- Let $\mathcal{S}$ be the collection of all $\mathcal{A}$ such that $\mathcal{A}$ has the following properties.

- $\mathcal{A}$ is a nonempty collection of strictly increasing sequences $s=\left\{s_{n}\right\}$ of positive integers.

- If $s \in \mathcal{A}$, then $s \gg b$, where $b=\left\{b_{n}\right\}$ is the sequence defined by $b_{n}:=n$ for all $n$.

- If $s, t \in \mathcal{A}$ and $s \neq t$, then $s \gg t$ or $t \gg s$.

If $u$ is the sequence defined by $u_{n}:=b_{n}^{2}$ for each $n$, then it is easy to see that $u \gg b$. It follows that the set $\mathcal{S}$ is nonempty. Order $\mathcal{S}$ by set-theoretic inclusion. By Zorn's Lemma, there exists a maximal element in $\mathcal{S}$.

Definition 2.3 Let $(\mathcal{Y}, \ll)$ be a totally ordered set and $B, C$ be subsets of $\mathcal{Y}$. We say that $B \ll C$ if $b \ll c$ for each $b \in B$ and each $c \in C$. A totally ordered set $(\mathcal{Y}, \ll)$ is an $\eta_{1}$-set if for any two countable subsets $B, C$ of $\mathcal{Y}$ such that $B \ll C$, there exists an element $a \in \mathcal{Y}$ such that $B \ll a \ll C$, i.e., $b \ll a \ll c$ for each $b \in B$ and each $c \in C$.

Let $\mathcal{A}$ be a maximal element in $\mathcal{S}$. This choice of $\mathcal{A}$ will be fixed through the rest of this section. For $s, t \in \mathcal{A}$, we define $s \ll t$ if and only if $s=t$ or $s \ll t$. Then $(\mathcal{A}, \ll)$ becomes a totally order set.

Theorem 2.4 ([11, Theorem 2.3]) The $\operatorname{set~}(\mathcal{A}, \lll)$ is an $\eta_{1}$-set.

\subsection{Chains of prime ideals in $D[[X]]$}

Suppose that $D$ is a non-SFT domain. Then there is an ideal $I$ of $D$ which is not SFT. As in $\left[1\right.$, p. 300], there exists a sequence $a_{0}, a_{1}, \ldots, a_{n}, \ldots$ of $I \subseteq D$ such that

$$
a_{m}^{m} \notin\left(a_{0}, a_{1}, \ldots, a_{m-1}\right)
$$

for each $m \geq 1$. Fix this sequence $a_{0}, a_{1}, \ldots, a_{n}, \ldots$. For an element $s=\left\{s_{n}\right\} \in \mathcal{A}$, let

$$
f_{s}:=a_{0}+a_{1} X^{s_{1}}+\cdots+a_{n} X^{s_{n}}+\cdots
$$

in $D[[X]]$. For elements $v_{1}, v_{2}, \ldots, v_{n}$ (not necessarily distinct) in $\mathcal{A}$, we let $I_{v_{1}, v_{2}, \ldots, v_{n}}$ be the ideal of $D[[X]]$ generated by all power series $f_{s}$ such that $s \gg v_{i}$ for each $i=1,2, \ldots, n$,

$$
I_{v_{1}, v_{2}, \ldots, v_{n}}:=\left\langle\left\{f_{s} \mid s \in \mathcal{A} \text { and } s \gg\left\{v_{1}, v_{2}, \ldots, v_{n}\right\}\right\}\right\rangle .
$$


Define

$$
\frac{I_{v_{1}, v_{2}, \ldots, v_{n}}}{f_{v_{1}} f_{v_{2}} \ldots f_{v_{n}}}:=\left\{\frac{f}{f_{v_{1}} f_{v_{2}} \ldots f_{v_{n}}} \mid f \in I_{v_{1}, v_{2}, \ldots, v_{n}}\right\}
$$

and

$$
S:=\bigcup_{\substack{n \in \mathbb{N} \\ v_{1}, v_{2}, \ldots, v_{n} \in \mathcal{A}}} \frac{I_{v_{1}, v_{2}, \ldots, v_{n}}}{f_{v_{1}} f_{v_{2}} \ldots f_{v_{n}}} .
$$

Then $S$ is a subset of the quotient field of $D[[X]]$. For elements $v_{1}, \ldots, v_{n}$ (not necessarily distinct) in $\mathcal{A}$, let $B=\left\{v_{1}, \ldots, v_{n}\right\}$ be a multiset. We also let $\|B\|:=n$. Define

$$
I_{B}:=I_{v_{1}, \ldots, v_{n}} \quad \text { and } \quad f_{B}:=\prod_{v \in B} f_{v}=f_{v_{1}} \ldots f_{v_{n}} .
$$

Then the set $S$ can be rewritten as

$$
S=\bigcup \frac{I_{B}}{f_{B}},
$$

where the union is taken over all multisets $B$ consisting of elements in $\mathcal{A}$. Let $D[[X]][S]$ be the ring generated by $S$ over $D[[X]]$. Denote by $S D[[X]][S]$ the ideal of $D[[X]][S]$ generated by $S$. Then this ideal is not the unit ideal ([11, Lemma 3.4]).

Theorem 2.5 ([6, Theorem 19.6]) Let $P$ be a prime ideal of an integral domain $R$. There exists a valuation overring $W$ of $R$ with maximal ideal $Q$ such that $Q \cap R=P$.

Let $R:=D[[X]][S]$. Since $S R$ is not the unit ideal of $R$, one can choose a prime ideal $P$ of $R$ such that $P$ contains $S R$. By Theorem 2.5, there exists a valuation overring $W$ of $R$ with maximal ideal $Q$ such that $Q \cap R=P$. For each $\alpha \in \mathcal{A}$, let $I_{\alpha} W$ be the ideal of $W$ generated by $I_{\alpha}$ and let

$$
P_{\alpha}:=\sqrt{I_{\alpha} W} \cap D[[X]] .
$$

Since $W$ is a valuation domain, $\sqrt{I_{\alpha} W}$ is a prime ideal of $W$. Hence, $P_{\alpha}$ is a prime ideal of $D[[X]]$. For $\alpha, \beta \in \mathcal{A}$, it can be shown that $\alpha \ll \beta$ if and only if $P_{\alpha} \supset P_{\beta}$. Hence, the set $\mathcal{P}:=\left\{P_{\alpha}\right\}_{\alpha \in \mathcal{A}}$ is a chain of prime ideals in $D[[X]]$. Furthermore, it is an $\eta_{1}$-set since $\mathcal{A}$ is.

A totally ordered set $A$ is Dedekind-complete provided that every nonempty subset of $A$ that has an upper bound has a supremum. Finally we can now present the main result of [11].

Theorem 2.6 If $D$ is a non-SFT domain, then $\operatorname{dim} D[[X]] \geq 2^{\aleph_{1}}$.

Proof. Let

$$
\mathcal{P}^{*}:=\left\{\cup_{\alpha \in \mathcal{D}} P_{\alpha} \mid \emptyset \neq \mathcal{D} \subseteq \mathcal{A}\right\} .
$$

Then $\mathcal{P}^{*}$ is a chain of prime ideals in $D[[X]]$ and $\mathcal{P}^{*}$ is Dedekind-complete. $\mathcal{P}^{*}$ contains an $\eta_{1}$-set, namely, $\mathcal{P}$. Therefore, by [5, Corollary 13.24$], \mathcal{P}^{*}$ has cardinality at least $2^{\boldsymbol{\aleph}_{1}}$.

\section{Transcendence degree in power series rings}

\subsection{Transcendence degree in polynomial rings}

Theorem 3.1 Let $D$ be an integral domain with quotient field $K$. Then $D[X]$ and $K[X]$ have the same quotient field. Hence,

$$
\operatorname{tr} . d .(K[X] / D[X])=1 .
$$

Proof. This is obvious. 


\subsection{Construction of an upper fathomless set $\mathcal{B}$}

In this subsection, we construct an uncountable set $\mathcal{B}$. In the next subsection, we will construct a set $\left\{\boldsymbol{a}_{f}\right\}_{f \in \mathcal{B}}$ of power series in $K[[x]]$ that is algebraically independent over the quotient field of $D[[x]]$ when $K[[x]]$ and $D[[x]]$ have different quotient fields.

Let $\mathbb{N}=\{1,2, \ldots\}$ be the set of positive integers. Set $\mathcal{S}$ be the collection of all functions $f: \mathbb{N} \rightarrow \mathbb{N}$ satisfying the following three conditions (see [18]).

- $f(i)>i$ for all $i \in \mathbb{N}$.

- $f(i+1)>f(i)+1$ for all $i \in \mathbb{N}$.

- For each $k \geq 1$, there exists an $I \geq 1$ (depending on $k$ ) such that $f(i+1)>k \cdot f(i)$ for all $i \geq I$.

The function $f(i)=2^{2^{i}}$ satisfies these three conditions. Hence, the set $\mathcal{S}$ is nonempty. For a function $f$ in $\mathcal{S}$, denote

$$
\operatorname{im}\left(\left.f\right|_{\geq I}\right)=\{f(i) \mid i \geq I\} .
$$

Hence, $\operatorname{im}\left(\left.f\right|_{\geq 1}\right)=\operatorname{im}(f)$ is the image of $f$.

Definition 3.2 For two functions $f$ and $g$ in $\mathcal{S}$, we define $g \gg f$ (we also write $f \ll g$ ) if there exists an integer $I \geq 1$ such that the following hold.

- $\operatorname{im}\left(\left.g\right|_{\geq I}\right) \subseteq \operatorname{im}(f)$ and $g(i)>f(i)$ for all $i \geq I$.

- If $s_{1}<s_{2}$ are both in $\operatorname{im}\left(\left.g\right|_{\geq I}\right)$, then they are not adjacent in $\operatorname{im}(f)$, i.e., there is no $i$ such that $s_{1}=f(i)$ and $s_{2}=f(i+1)$.

Remark 3.3 For $f, g, h \in \mathcal{S}$, if $f \ll g$ and $g \ll h$, then $f \ll h$.

Let $C(\mathcal{S})$ denote the collection of all nonempty subsets $\mathcal{B}$ of $\mathcal{S}$ satisfying: for any two different functions $f$ and $g$ in $\mathcal{B}$, either $f \gg g$ or $g \gg f$. We order $C(\mathcal{S})$ by set-theoretic inclusion. By Zorn's Lemma, there exists a maximal element in $C(\mathcal{S})$. Let $\mathcal{B}$ be a maximal element in $C(\mathcal{S})$. This choice of $\mathcal{B}$ will be fixed through the rest of the section. For $f, g \in \mathcal{B}$, we define $f \ll g$ if and only if $f=g$ or $f \ll g$. Then $(\mathcal{B}, \ll)$ becomes a totally ordered set.

Definition 3.4 A totally ordered set $(\mathcal{Y}, \ll)$ is called an upper fathomless set if for every nonempty countable subset $C$ of $\mathcal{Y}$, there exists an element $y \in \mathcal{Y}$ such that $y \gg c$ for all $c \in C$.

By definition, every upper fathomless set is an uncountable set. The following is [8, Theorem 7].

Theorem 3.5 The set $(\mathcal{B}, \ll)$ is an upper fathomless set and hence is uncountable.

\subsection{Transcendence degree in power series rings}

Suppose that $D$ is an integral domain with quotient field $K$ such that the quotient field of $K[[X]]$ properly contains the quotient field of $D[[X]]$. Then by $[7$, Theorem 1], there exists a sequence $\left\{a_{i}\right\}_{i=1}^{\infty}$ of nonzero elements of $D$ such that $\cap_{i=1}^{\infty}\left(a_{i}\right)=(0)$. Replacing $\left\{\left(a_{i}\right)\right\}_{i=1}^{\infty}$ by a (suitable) strictly descending subsequence if necessary, we may assume that the sequence $\left\{\left(a_{i}\right)\right\}_{i=1}^{\infty}$ is strictly descending (see [7, Remark 1]), i.e.,

$$
\left(a_{1}\right) \supset\left(a_{2}\right) \supset \cdots \supset\left(a_{i}\right) \supset \cdots .
$$


Therefore, if $i_{1} \geq i_{2}$ then $\left(a_{i_{1}}\right) \subseteq\left(a_{i_{2}}\right)$ and hence $a_{i_{1}}$ is a multiple of $a_{i_{2}}$. Let $\mathcal{B}$ be the upper fathomless set constructed in Subsection 3.2. For each $f \in \mathcal{B}$, define a power series $\boldsymbol{a}_{f}$ in $K[[X]]$ by

$$
\boldsymbol{a}_{f}=\sum_{i=1}^{\infty}\left(X / a_{i}\right)^{f(i)} .
$$

We will give sketch of proof of the following main result of the section. For a power series $\boldsymbol{p}$ in $K[[X]]$, the support of $\boldsymbol{p}$, denoted by $\operatorname{supp}(\boldsymbol{p})$, is the set of all nonnegative integer $s$ for which the coefficient of $X^{s}$ in $\boldsymbol{p}$ is nonzero.

Theorem 3.6 ([8, Theorem 12]) Suppose that D is an integral domain with quotient field $K$ such that $D[[X]]$ and $K[[X]]$ have different quotient fields. Then the quotient field of $K[[X]]$ has uncountable transcendence degree over the quotient field of $D[[X]]$, i.e, t.r. $(K[[X]] / D[[X]]) \geq \boldsymbol{\aleph}_{1}$.

Sketch of proof. We show that the set $\left\{\boldsymbol{a}_{f}\right\}_{f \in \mathcal{B}}$ is algebraically independent over the quotient field of $D[[X]]$. Suppose on the contrary that there are some $f_{1}, f_{2}, \ldots, f_{J} \in \mathcal{B}$ such that $\boldsymbol{a}_{f_{1}}, \boldsymbol{a}_{f_{2}}, \ldots, \boldsymbol{a}_{f_{J}}$ are algebraically dependent over the quotient field of $D[[X]]$. We may assume that

$$
f_{1} \ll f_{2} \ll \cdots \ll f_{J} .
$$

Let $P\left(t_{1}, \ldots, t_{J}\right)$ be a nonzero polynomial with coefficients in the quotient field of $D[[X]]$ such that

$$
P\left(\boldsymbol{a}_{f_{1}}, \ldots, \boldsymbol{a}_{f_{J}}\right)=0 .
$$

Multiplying both sides of this equation by a suitable element in $D[[X]]$, we may assume that the coefficients of $P\left(t_{1}, \ldots, t_{J}\right)$ are in $D[[X]]$. Let $M$ be the set of all power series of the form $\boldsymbol{a}_{f_{1}}^{e(1)} \ldots \boldsymbol{a}_{f_{J}}^{e(J)}$ corresponding to those monomials $t_{1}^{e(1)} \ldots t_{J}^{e(J)}$ which occur in $P\left(t_{1}, \ldots, t_{J}\right)$ with nonzero coefficient. Then the relation $P\left(\boldsymbol{a}_{f_{1}}, \ldots, \boldsymbol{a}_{f_{J}}\right)=0$ is actually a linear combination of the elements of $M$ with coefficients in $D[[X]]$. Order the elements of $M$ lexicographically, using the exponents of $\boldsymbol{a}_{f_{1}}$, then $\boldsymbol{a}_{f_{2}}$, and so forth. Write $M=\left\{\boldsymbol{m}_{0}, \boldsymbol{m}_{\mathbf{1}}, \ldots, \boldsymbol{m}_{R}\right\}$, where $\boldsymbol{m}_{0}$ is the largest term in this ordering. Then $P\left(\boldsymbol{a}_{f_{1}}, \ldots, \boldsymbol{a}_{f_{J}}\right)=0$ has the form

$$
\boldsymbol{d}_{0} \boldsymbol{m}_{0}+\boldsymbol{d}_{1} \boldsymbol{m}_{1}+\cdots+\boldsymbol{d}_{R} \boldsymbol{m}_{R}=0
$$

for some $\boldsymbol{d}_{r} \in D[[X]](r=0,1, \ldots, R), \boldsymbol{d}_{0} \neq 0$. Denote by $k$ the index of the first nonzero coefficient $d_{0, k}$ of $\boldsymbol{d}_{0}$. The proof is finished by showing a contradiction that $0 \neq d_{0, k} \in \cap_{i=1}^{\infty}\left(a_{i}\right)$, which is technically complicated and is omitted.

\section{$4 \boldsymbol{\aleph}_{0}$-generated power series rings}

The purpose of this section is to show that the power series ring $R \llbracket X \rrbracket$ is an $\boldsymbol{\aleph}_{0}$-generated ring if and only if $R \llbracket X \rrbracket$ is a Noetherian ring (and hence) if and only if $R$ is a Noetherian ring. In order to prove this result, we only need to show that if $R$ is a non-Noetherian ring, then the power series ring $R \llbracket X \rrbracket$ is not an $\boldsymbol{\aleph}_{0}$-generated ring since if $R$ is a Noetherian ring, then $R \llbracket X \rrbracket$ is also a Noetherian ring (for example, see [13, Theorem 71]) and hence an $\boldsymbol{\aleph}_{0}$-generated ring. Suppose that $R$ is a non-Noetherian ring. Our task is to construct an ideal $J$ of $R \llbracket X \rrbracket$ that cannot be generated by any countable subset of $J$. Using the $\eta_{1}$-set $(\mathcal{A}, \ll)$ in Section 2 , we construct generators for the $J$ of $R \llbracket X \rrbracket$ as follows.

Let $R$ be a non-Noetherian ring. Then there exists a sequence $a_{0}, a_{1}, \ldots, a_{m}, \ldots$ of elements in $R$ such that

$$
a_{m} \notin\left(a_{0}, a_{1}, \ldots, a_{m-1}\right)
$$


for each $m \geq 1$. For each integer $m \geq 0$, we let $I_{m}:=\left(a_{0}, a_{1}, \ldots, a_{m}\right)$. Then $a_{m} \notin I_{m-1}$ for each $m \geq 1$. For each sequence $s=\left\{s_{n}\right\} \in \mathcal{A}$, we define

$$
f_{s}:=a_{0}+a_{1} X^{s_{1}}+a_{2} X^{s_{2}}+\cdots+a_{n} X^{s_{n}}+\cdots \in R \llbracket X \rrbracket
$$

We let $J$ be the ideal of $R \llbracket X \rrbracket$ generated by all $f_{s}$ with $s \in \mathcal{A}$. The following is the main result of [10].

Theorem 4.1 ([10, Theorem 13]) For a ring $R$, the following are equivalent.

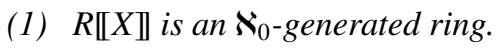

(2) $R \llbracket X \rrbracket$ is a Noetherian ring.

(3) $R$ is a Noetherian ring.

Proof. We only need to prove that (1) implies (3). Suppose that $R$ is not a non-Noetherian ring. We show that $R \llbracket X \rrbracket$ is not an $\boldsymbol{\aleph}_{0}$-generated ring. It suffices to show that the ideal $J$ constructed above is not a countably generated ideal. Suppose on the contrary that $J$ is countably generated. Then there exists a countable subset $\mathcal{B}$ of $\mathcal{A}$ such that $J$ is generated by $\left\{f_{s} \mid s \in \mathcal{B}\right\}$. Since $\mathcal{A}$ is a fathomless set, there exists a sequence $v \in \mathcal{A}$ such that $v \ll \mathcal{B}$. Since $f_{v} \in J, f_{v}$ is a finite sum of elements of the form $h(s) f_{s}$,

$$
f_{v}=\sum_{s} h(s) f_{s}
$$

where $h(s) \in R \llbracket X \rrbracket$ and $s \in \mathcal{B}$. Since $v \ll \mathcal{B}$, by taking a finite intersection of members of $\mathcal{U}$, we can find a set $U \in \mathcal{U}$ such that $v_{m}<s_{m}$ for each $m \in U$ and for each $s$ appearing in the finite sum (1). Choose any number $m \in U$. Since $v_{m}<s_{m}$, the coefficient of $f_{s}$ at $X^{j}$ belongs to $I_{m-1}$ for all $j \leq v_{m}$. It follows that the coefficient of $h(s) f_{s}$ at $X^{v_{m}}$ belongs to $I_{m-1}$. This holds for every $s$ appearing in the finite sum (1). Therefore, the coefficient of $\sum_{s} h(s) f_{s}$ at $X^{v_{m}}$ belongs to $I_{m-1}$. This is a contradiction since the coefficient of $f_{v}=\sum_{s} h(s) f_{s}$ at $X^{v_{m}}$ is $a_{m}$ and $a_{m} \notin I_{m-1}$.

Corollary 4.2 If $R$ is an $\aleph_{0}$-generated ring, then the power series ring $R \llbracket X \rrbracket$ is not necessarily an $\boldsymbol{\aleph}_{0}$-generated ring.

Proof. Let $R$ be any $\boldsymbol{\aleph}_{0}$-generated ring which is not a Noetherian ring (for example, let $R=\mathbb{Q}\left[X_{1}, X_{2}, \ldots, X_{n}, \ldots\right]$, the polynomial ring in countably infinite indeterminates over the field of rational numbers $\mathbb{Q}$ ). Then $R \llbracket X \rrbracket$ is not an $\boldsymbol{\aleph}_{0}$-generated ring by Theorem 4.1.

The above corollary contrasts with the following famous result for polynomial rings.

Theorem 4.3 (Hilbert Basis Theorem.) If $R$ is a Noetherian ring, then so is the polynomial ring $R[X]$.

In comparing with Theorem 4.1, on has the following result, whose its proof follows the standard one of Hilbert Basis Theorem.

Theorem 4.4 ([10, Theorem 22]) For any infinite cardinal number $\alpha$, a ring $R$ is an $\alpha$ generated ring if and only if the polynomial ring $R[X]$ is an $\alpha$-generated ring. 


\section{Conclusion}

Being the completion of the polynomial ring $R[X]$, the power series ring $R[[X]]$ however has very strange behaviors. The paper presents the following three examples.

- If $\operatorname{dim} R$ is finite, then so is $\operatorname{dim} R[X]$. However, $\operatorname{dim} R[[X]]$ can be infinite, in fact $\geq 2^{\aleph_{1}}$, even if $\operatorname{dim} R$ is finite.

- If $D$ is an integral domain with quotient field $K$, then $D[X]$ and $K[X]$ share the same quotient field. However, the quotient field of $K[[X]]$ is much larger than that of $D[[X]]$. In fact, the quotient field of $K[[X]]$ is often has uncountable transcendence degree over that of $D[[X]]$.

- The concepts "Noetherian ring" and " $\boldsymbol{\aleph}_{0}$-generated ring" are the same for the power series ring $R[[X]]$ while they are different for the polynomial ring $R[X]$. In fact, the polynomial ring $R[X]$ is an $\alpha$-generated ring if and only if $R$ is an $\alpha$-generated ring, which is an analogue of Hilbert Basis Theorem stating that the polynomial ring $R[X]$ is a Noetherian ring if and only if $R$ is a Noetherian ring.

Techniques involved in the proofs of the above results are quite complicated. By exploring these, we hope that similar techniques can be further developed to have a better understand of the power series ring $R[[X]]$.

\section{References}

[1] J.T. Arnold, Krull dimension in power series rings, Trans. Amer. Math. Soc. 177, 299304 (1973).

[2] J.T. Arnold and D.W. Boyd, Transcendence degree in power series rings, J. Algebra 57, no. 1, 180-195 (1979).

[3] J.W. Brewer, Power series over commutative rings, Lecture Notes in Pure Appl. Math. 64, (Marcel Dekker, Inc., New York, 1981).

[4] R. Gilbert, A note on the quotient field of the domain $D[[X]]$, Proc. Amer. Math. Soc. 18, 1138-1140 (1967).

[5] L. Gillman and M. Jerison, Rings of continuous functions, (The University Series in Higher Mathematics, D. Van Nostrand Co., Inc., Princeton, N.J.-Toronto-London-New York, 1960).

[6] R. Gilmer, Multiplicative ideal theory, (Marcel Dekker, New York, 1972).

[7] R. Gilmer, On commutative rings of finite rank, Duke Math. J. 39, 381-383 (1972).

[8] L.T.N. Giau and P.T. Toan, Transcendental Degree in Power Series Rings, J. Algebra 501, 51-67 (2018).

[9] B.G. Kang and M.H. Park, Krull-dimension of the power series ring over a nondiscrete valuation domain is uncountable, J. Algebra 378, 12-21 (2013).

[10] B.G. Kang and P.T. Toan, A remark on the Noetherian property of power series rings Pacific J. Math. 283, 353-363 (2016).

[11] B.G. Kang and P.T. Toan, Krull dimension of power series rings over non-SFT domains, J. Algebra 499, 516-537 (2018).

[12] B.G. Kang and P.T. Toan, Krull dimension of a power series ring over a valuation domain, preprint.

[13] I. Kaplansky, Commutative rings, Revised edition, (The University of Chicago Press, Chicago, Ill.-London, 1974).

[14] K.A. Loper and T.G. Lucas, Constructing chains of primes in power series rings, J. Algebra 334, 175-194 (2011). 
[15] K.A. Loper and T.G. Lucas, Constructing chains of primes in power series rings II, J. Algebra Appl. 12, no. 1, 1250123, 30 pp. (2013).

[16] A. Seidenberg, A note on the dimension theory of rings, Pacific J. Math. 3, 505-512 (1953).

[17] A. Seidenberg, On the dimension theory of rings II, Pacific J. Math. 4, 603-614 (1954).

[18] P.B. Sheldon, How changing $D[[X]]$ changes its quotient field, Trans. Amer. Math. Soc. 159, 223-244 (1971). 